\title{
Characterization of Roselle (Hibiscus sabdariffa L. var. altissima Wester) Accessions in Northern Ghana by Agro-morphological Traits
}

\author{
Nii Ayi Ankrah ${ }^{1}$, Antonia Y. Tetteh ${ }^{1}$, Nancy Coffie ${ }^{1} \&$ Achana Niagiah $^{1}$ \\ ${ }^{1}$ Department of Biochemistry and Biotechnology, Kwame Nkrumah University of Science and Technology, \\ Kumasi, Ghana \\ Correspondence: Antonia Y. Tetteh, Department of Biochemistry and Biotechnology, Kwame Nkrumah \\ University of Science and Technology, Kumasi, Ghana. Tel: 233-(020)-113-4416. E-mail: \\ atetteh.sci@knust.edu.gh; aytetteh@gmail.com
}

Received: March 6, 2018

doi:10.5539/jas.v10n9p64

\author{
Accepted: June 7, $2018 \quad$ Online Published: August 15, 2018 \\ URL: https://doi.org/10.5539/jas.v10n9p64
}

\begin{abstract}
Roselle (Hibiscus sabdariffa L. var. altissima Wester) is a bast fiber crop of global economic importance. Although West Africa is considered the centre of diversity, roselle research and utilization is widely ignored. The awakening of industrialization in Ghana presents roselle as a candidate crop for exploration, however, information on genotypes of economic importance is lacking. Our objective was to map roselle population hotspots in northern Ghana and examine genetic variability therein. Thirty-six roselle accessions collected from five regions in Ghana were planted in field trials using a $6 \times 6$ lattice square design in three replications and evaluated for seven qualitative and four quantitative morphological traits covering plant type, leaf and stem characteristics, and growth habit. Data were analysed by Shannon-Weiner Diversity Index (SDI) and analysis of variance. A large variability was identified between the accessions. The mean SDI values in the 18 districts ranged from 0.53 to 0.73 with Savelugu-Nanton district having the largest diversity and having accessions with the highest mean plant height of $308.27 \pm 48.91 \mathrm{~cm}$, highest branching point at $107.19 \pm 64.66 \mathrm{~cm}$, and few branches not exceeding 5.0 in number. Majority of the accessions exhibited low branching points. The most variable trait was branch number with SDI of $0.83 \pm 0.12$. Accessions HA-07, HA-11, HA-12, HA-21, and HA-33 ranked highest with respect to plant height with few branches at high branching points, and large basal diameter. The ample diversity in roselle and identification of genotypes of economic importance await their exploitation for genetic improvement, particularly for fiber yield.
\end{abstract}

Keywords: roselle, variability, genetic diversity, morphology

\section{Introduction}

Roselle (Hibiscus sabdariffa L. var. altissima Wester), the inedible calyx type of $H$. sabdariffa species is of the Malvaceae family which includes other fiber crops such as cotton (Gossypium hirsutum L.), jute (Corchorus capsularis L. and Corchorus olitorius L.), and kenaf (Hibiscus cannabinus L.). Roselle is believed to be native to Africa (Boulanger et al., 1984; Gomez-Leyva et al., 2008). A recent study of roselle collection from West Africa, specifically, Senegal to Nigeria, revealed that the region between northern Ghana to Mali through Burkina Faso is a center of rich diversity of roselle (Coffie, 2016). In this region, roselle grows in the wild and sprouts after the first rains. Here, roselle is known severally owing to the array of morphotypes available. While roselle is generally called sobooro or suure, indigenous information lists over 25 names for roselle among the tribal folks indicating a historical evidence of its domestication in northern Ghana. Some common names are "biito" among the Nankana and Frafra, "vio" in Grushi and Kasem, and "tingyanbam" in Konkomba. Together with its closely related variety, $H$. sabdariffa var. sabdariffa, which is principally grown for its edible calyx, both crops are under homestead cultivation by the traditional small-scale farmers in northern Ghana where their leaves, fibers and seeds are used for nutritional and medicinal purposes (Perry, 1980; Morton, 1987; Rhodin \& Panchoo, 1990) and for tethering livestock.

Records indicate that roselle was accidentally carried from Gold Coast to the Philippines in 1911 where it was cultivated and studied (Wester, 1912, 1914; Andrews, 1952). Roselle variety specimens were also carried from Northern territories of Gold Coast (Ghana) to Kew Gardens for identification in 1912 (Imperial Institute, 1912). 
The current stringent environmental regulations and restrictions on synthetic fibers have led to increase in demand for roselle as source of bast fiber for upholstery and sac making, for paper pulp, as a salt-resistant cordage, and as composite for electronic and building materials. In Ghana, roselle use is limited to culinary aspects for its leaves and seeds.

Current interest in roselle fiber for commercial cultivation in Ghana necessitates collection, evaluation, genetic improvement and conservation, however, scientific information on characterization of roselle types in Ghana is limited. An agro-morphological characterization and molecular profiling by Random Amplified Polymorphic DNA (RAPD) markers of roselle collection in Ghana contained only three altissima genotypes, the rest being sabdariffa types (Coffie, 2016). This study revealed that contrary to reports of lack of variability in exotic genotypes of roselle to merit success with genetic improvement (Hanboonsong et al., 2000; Yusof \& Saud, 2009; Omalsaad et al., 2014), a large variability existed in the West African sabdariffa genotypes (Coffie, 2016).

Efficient utilization of a crop is largely dependent on the nature and magnitude of its diversity for genetic improvement in yield, disease resistance, and quality characteristics (Dudley \& Moll, 1969; Chand et al., 2008). Agro-morphological characterization is a common system for estimation of genetic diversity as it uncovers phenotypic differences, relationships among genotypes, and evolutionary history of the crop. Genetic diversity estimation by phenotypic characterization encompasses estimation of both within and between population variations. Within-population diversity estimates the number of unique classes within a population as richness, and the relative abundance of the genotypes within the classes as evenness. A population with large number of diverse genotypes that are equally distributed will have high richness diversity index, whereas that with few diverse genotypic groups will have low diversity index (Brower \& Zar, 1977). Four most common estimates that measure diversity as a composite of the two assessments include Berger-Parker index (Berger \& Parker, 1970), McIntosh (1967), Simpson (1949), and Shannon-Weiner diversity index (SDI) (Shannon \& Weaver, 1949). Simpson's index, which predominantly estimates evenness, relies on the few most abundant species to estimate diversity without considering the rare species, (Risser \& Rice, 1971; Loya, 1972; Whittaker, 1972). Berger-Parker Index has been used extensively to establish the more abundant species within a community and estimates species richness (May 1975). Shannon's diversity combines both richness and evenness but is influenced by the number of species (Pielou, 1969). DeJong (1975) reported that the McIntosh diversity index overcomes the sample size effect of Shannon's and estimates diversity as a percentage of the maximum possible diversity attainable by that sample size. The standardized Shannon diversity index which ranges from 0 to 1.0 is more useful as it allows for comparison of accessions on the same scale. Medagam et al. (2015) reported SDI of Indian vegetable roselle $(H$. sabdariffa var. sabdariffa) for plant characteristics, leaf yield, and total biomass as 0.358 to 2.00 . To the best of our knowledge, the diversity and genetic variability within the roselle fiber types in Ghana has not been assessed. Similarly, the distribution of variation in roselle populations in the northern sector of Ghana where roselle originates is not known. We report the genetic variation in roselle fiber types in a northern Ghana collection assessed by morphological traits.

\section{Materials and Methods}

\subsection{Plant Material}

Seeds of thirty-six accessions of roselle, designated HA-01 to HA-36 were collected from mature naturally growing wild plants on farmers' field in 18 districts covering five regions of Ghana at longitude $0.24^{\circ} \mathrm{W}$ in Bawku to $2.51^{\circ} \mathrm{W}$ in Wa and latitude $6.66^{\circ} \mathrm{N}$ in Kumasi to $11.05^{\circ} \mathrm{N}$ in Bawku. The elevations covered 132 to 408 m.a.s.l. (Figure 1). Seed collection was guided by farmers' indigenous knowledge. Seeds were planted from July to December 2016 on the research fields of the Department Horticulture, Kwame Nkrumah University of Science and Technology, Kumasi, located at longitude $1.41^{\circ} \mathrm{W}$ to $1.60^{\circ} \mathrm{W}$ and latitude $6.71^{\circ} \mathrm{N}$ to $6.75^{\circ} \mathrm{N}$. The experiment design was a $6 \times 6$ lattice square with three replications. Plot size was $0.5 \mathrm{~m} \times 2.0 \mathrm{~m}$ with plant spacing of $25 \mathrm{~cm} \times 50 \mathrm{~cm}$ separated by an alley of $0.5 \mathrm{~m}$ to give a plant density of 150,000 plants/ha. Irrigation was carried out as and when needed. Pre-emergence weeds were controlled with Round-Up Ready (glyphosate, $360 \mathrm{~g} / \mathrm{L}$ ) at a rate of $3.5 \mathrm{~L} / \mathrm{ha}$. Post-emergence weeds were cleared from the field by hand weeding using a hoe. The predominant insect pests, cotton stainers (Dysdercus sp.), cabbage fly (Delia radium), and thrips (Thysanoptera) were controlled with Sumitex (dimethoate, $400 \mathrm{~g} / \mathrm{L}$ ) applied at a rate of $1 \mathrm{~L} / \mathrm{ha}$.

\subsection{Data Collection}

At 120 days after planting, data were collected on 7-10 competitive plants per plot. Six qualitative traits on leaf, stem, and growth habit, and four quantitative traits, namely, plant height, height at first branching, branch number, and basal diameter were recorded. Leaf colour was evaluated as the predominant colour of the leaf blade (uniformly green $=1$, pigmented $=2$ ); and leaf size was recorded as appearance of width of leaf $($ slender $=$ 
1 , broad $=3$ ). Roselle leaves differentiate progressively with maturation such that more than one leaf form can occur on one plant, hence leaf form was rated as the predominant shape of leaf in the mid-portion of the plant (entire $=1$, tri-lobed $=3$, penta-lobed $=5$ ). Plant type was scored as the predominant colour of the stem (uniformly green $=1$, pigmented $=3$, uniformly red $=5$ ); branching habit was recorded as the form of branching ( few $=1$, intermediate $=2$, extensive $=3$ ); stem pubescence was determined as evenness of surface of stem ( smooth $=1$, others $=3$ ), and branching height was measured as position of the branches with respect to the height of the plant (low and close to base $=1$, branching around the middle or intermediate $=3$, branching around the apex or high branching $=5$ ). For the quantitative traits, plant height was measured as height from ground level to the growing tip in $\mathrm{cm}$, height at first branching was measured from ground level to first branch in $\mathrm{cm}$ using a meter rule. The basal diameter was measured at $5 \mathrm{~cm}$ above the ground using a micrometer screw gauge. The number of branches on a plant was scored as branch number.

\subsection{Data Analysis}

Frequencies were calculated for the qualitative data. For the quantitative data, means, standard deviations, minimum and maximum values, and coefficient of variation were computed, Analyses of variance were computed to estimate variation between population. The within population variation was assessed by the standardized Shannon-Wiener Diversity Index,

$$
H^{\prime}=\frac{-\sum(P i \cdot \log P i)}{\log n}
$$

where, $P_{i}$ is the fraction of the entire species population, $n$ is the number of classes with observations, and $\log n$ $=$ logarithm of $n$. For each trait, the population was subdivided into classes on the basis of the mean $(\mathrm{Y})$ and standard deviation $(\sigma)$ into six classes in the range of $(\mathrm{Y}-2 \sigma)>\mathrm{Y}>(\mathrm{Y}+2 \sigma)$ where $Y$ is response for each accession trait. The mean diversity index of accessions in each district was calculated. All computations were carried out in SAS 9.3 software (SAS Institute, 2011).

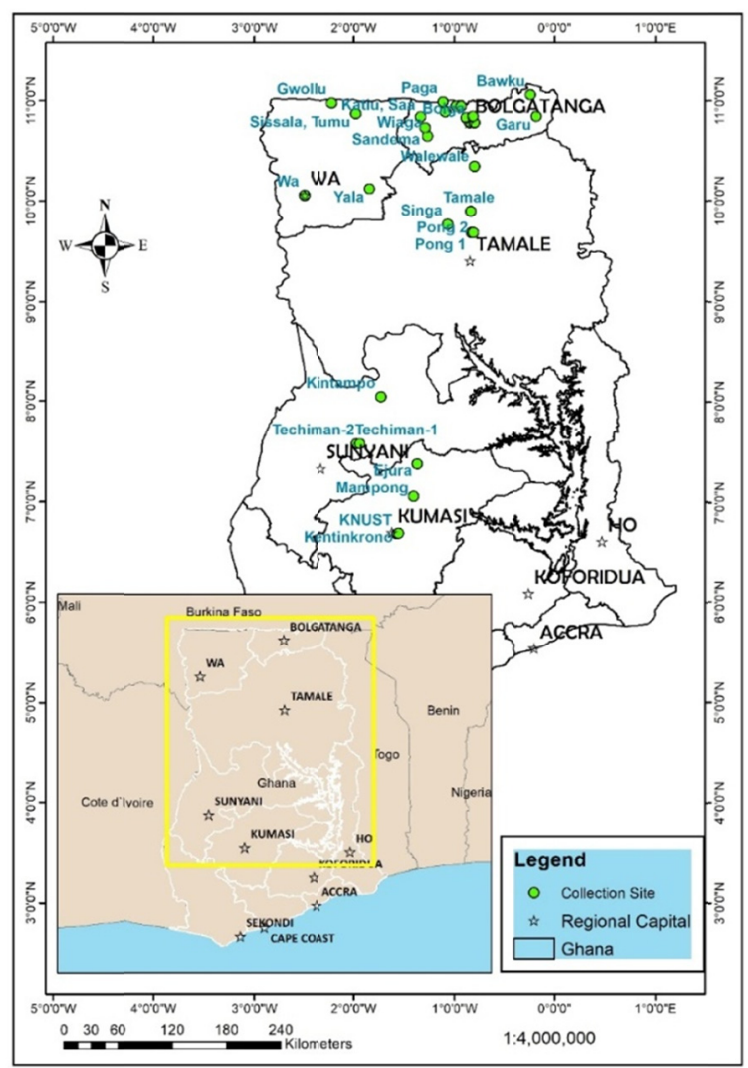

Figure 1. A schematic map of Ghana showing roselle collection sites from Kumasi (Ashanti) to Bolgatanga (Upper East) and Wa (Upper West) 


\section{Results}

\subsection{Variation in Plant Characteristics}

The within and between population variation in 36 accessions of roselle fiber type collected from 18 districts in Ghana were investigated by morphological trait assessment in a field study in Kumasi, Ghana. Bolgatanga district was represented by seven accessions (19.44\%), Kassena-Nankana by five accessions (13.89\%), Kumasi and Savelugu-Nanton districts had three (8.33\%) accessions each, Ejura-Sekyedumase, Sissala, Tamale, and Techiman districts were represented by two (5.56\%) accessions each, while Bawku, Ejisu-Juaben, Garu-Tempane, Gowri-Yorogo, Kintampo, Mampong Sagnarigu-Garu, Talensi, Wa and West Mamprusi had one $(2.78 \%)$ accession each.

In all, 703 plants were evaluated. Accessions exhibited ample variability in, leaf shape, plant type, and branching habit as they had three distinct categories of plant types. In contrast, leaf colour, leaf size, and stem pubescence were uniform and less variable (Table 1). The distribution of plant characteristics were $423(63 \%)$ uniformly green, 180 (27\%) pigmented, and $71(10 \%)$ uniformly red, $396(59 \%)$ plants with few branches, $238(36 \%)$ with intermediate branching, and $33(5 \%)$ plants with extensive branching. Branching height was less variable with $547(82 \%)$ having low branching points, $71(11 \%)$ with intermediate branching, and 48 plants $(7 \%)$ with high branching points. Stem pubescence was less variable. Majority of the plants $(78 \%)$ were hairy, rough, or prickly, and $22 \%$ had smooth stems and leaves. Variation in leaf colour and leaf size was low. A large number, 646 plants (96\%) had green leaves, and 28 (4\%) were pigmented. Regarding leaf size, majority of the plants $639(95 \%)$ had slender leaves and $35(5 \%)$ had broad leaves. Ample variability was present in leaf shape with $456(68 \%)$ being tri-lobed, 139 (21\%) were penta-lobed, and 79 plants (12\%) had entire leaves.

Table 1. Distribution of qualitative morphological traits in $H$. sabdariffa L. var. altissima collected from Northern Ghana and evaluated in 2016

\begin{tabular}{|c|c|c|c|c|}
\hline Trait & Description & Score & Distribution & Percentage (\%) \\
\hline \multirow[t]{3}{*}{ Plant type } & Uniformly green & 1 & 423 & 62.67 \\
\hline & Pigmented & 3 & 180 & 26.71 \\
\hline & Uniformly red & 5 & 71 & 10.53 \\
\hline \multirow[t]{3}{*}{ Branching habit } & Few & 1 & 396 & 59.37 \\
\hline & Intermediate & 2 & 238 & 35.68 \\
\hline & Extensive & 3 & 33 & 4.95 \\
\hline \multirow[t]{3}{*}{ Branching Height } & Low & 1 & 547 & 82.13 \\
\hline & Intermediate & 3 & 71 & 10.66 \\
\hline & High & 5 & 48 & 7.21 \\
\hline \multirow[t]{2}{*}{ Stem pubescence } & Smooth & 1 & 146 & 21.66 \\
\hline & Other & 3 & 528 & 78.34 \\
\hline \multirow[t]{2}{*}{ Leaf colour } & Uniformly Green & 1 & 646 & 95.85 \\
\hline & Pigmented & 2 & 28 & 4.15 \\
\hline \multirow[t]{2}{*}{ Leaf size } & Slender & 1 & 639 & 94.81 \\
\hline & Broad & 3 & 35 & 5.19 \\
\hline \multirow[t]{3}{*}{ Leaf shape } & Entire & 1 & 79 & 11.72 \\
\hline & 3-lobed & 3 & 456 & 67.66 \\
\hline & 5-lobed & 5 & 139 & 20.62 \\
\hline
\end{tabular}

\subsection{Variation in Quantitative Traits}

Mean squares of genotypes were highly significant $(\mathrm{P}<0.01)$ for three traits, namely, plant height, height at first branching, and branch number. Basal diameter was also significantly different $(\mathrm{P}<0.05)$ among the genotypes. Based on the coefficient of variation, height at first branching (16.39\%) and branch number $(25.47 \%)$ had more widely dispersed values than plant height $(4.48 \%)$ and basal diameter $(10.81 \%)$ (Table 2). Plant height and height at first branching of the accessions were significantly different $(\mathrm{P}<0.05)$ from one district to another, whereas branch number and basal diameter were not different (Table 3). 


\subsection{Variability in Districts Accessions}

Variability in the accessions from different districts was remarkable. Analysis of variance for the district data produced highly significant $(\mathrm{P}<0.001)$ mean squares for plant height, height at first branching, branch number and basal diameter (Table 3).

Overall mean plant height for the populations was $240.69 \pm 23.22 \mathrm{~cm}$ and ranged from 164 to $389 \mathrm{~cm}$. On the basis of mean of traits at the districts, plant height varied from $214.86 \pm 19.72 \mathrm{~cm}$ in Gowri-Yorogo (Upper East) to $308.27 \pm 48.91 \mathrm{~cm}$ in Savelugu-Nanton (Northern). Majority of the districts exhibited low values of height at first branching with a mean of $25.20 \pm 25.97 \mathrm{~cm}$ and range of 1.2 to $215 \mathrm{~cm}$. Again, Kintampo Municipal (Brong Ahafo) accessions exhibited the least height at first branching of $10.80 \pm 4.56 \mathrm{~cm}$ and Savelugu-Nanton (Northern) had the highest value of $107.39 \pm 64.66 \mathrm{~cm}$. Overall mean of branch number of 5.64 \pm 1.16 varied from 1.0 to 17 . Based on district means, values ranged from 3.76 \pm 1.73 in Ejisu-Juaben (Ashanti) to $8.27 \pm 2.10$ in Sagnarigu-Gariba (Upper East). The basal diameter had an overall mean of $18.52 \pm 2.05 \mathrm{~mm}$ and ranged from $10.12 \mathrm{~mm}$ to $31.34 \mathrm{~mm}$. The district means of these traits ranged from a minimum value of $13.51 \pm 3.10 \mathrm{~mm}$ in the Mampong Municipal (Ashanti) to a maximum of 21.28 $44.60 \mathrm{~mm}$ in the Kassena-Nankana (Upper East) (Table 3). Accessions from four districts consistently had tall plants exceeding $250.0 \mathrm{~cm}$. These sites were Bolgatanga Municipal $(251.46 \pm 41.72 \mathrm{~cm})$, Kassena-Nankana $(266.30 \pm 58.94 \mathrm{~cm})$, Savelugu-Nanton $(308.27 \pm 48.91 \mathrm{~cm})$, and Techiman Municipal $(287.26 \pm 58.26 \mathrm{~cm})$. Similarly, accessions from these districts showed intermediate branching points of $33.63 \pm 47.25 \mathrm{~cm}, 45.00 \pm 54.78 \mathrm{~cm}, 107.19 \pm 64.66 \mathrm{~cm}$ and $88.84 \pm 75.97 \mathrm{~cm}$ respectively. Number of branches per plant ranged from three to ten. Districts having least number of branches of three to four were Ejisu-Juaben (3.76 \pm 1.73$)$, Ejura-Sekyedumase (4.42 \pm 1.86 ), Mampong Municipal (4.25 \pm 2.43$)$, Savelugu-Nanton (4.69 \pm 2.03$)$, Sissala (4.81 \pm 3.79$)$, Talensi (4.88 \pm 3.33$)$, Techiman Municipal (4.61 \pm 2.65$)$, and Wa Municipal $(4.42 \pm 1.74)$ accessions. Mean basal diameter at the various districts ranged from $13.51 \pm 3.1 \mathrm{~mm}$ in Mampong Municipal to $21.28 \pm 4.60 \mathrm{~mm}$ in Kassena-Nankana accessions. Values exceeding $20 \mathrm{~mm}$ were found in accessions of Bolgatanga Municipal 20.61 $\pm 2.37 \mathrm{~mm}$, Kassena-Nankana 21.28 $\pm 4.60 \mathrm{~mm}$, and Savelugu-Nanton $20.06 \pm 2.30$ $\mathrm{mm}$ (Table 3).

Table 2. Mean squares of quantitative triats in roselle evaluated in field trial in Ghana in 2016

\begin{tabular}{llllll}
\hline \multirow{2}{*}{ Source of variation } & \multirow{2}{*}{ df } & \multicolumn{3}{c}{ Mean squares } \\
\cline { 3 - 6 } & & Plant height & Height at first branching & Branch number & Basal diameter \\
\hline Block (Replication) & 15 & 141.09 & 21.84 & 1.76 & 3.72 \\
Genotype & 35 & $3778.02^{* *}$ & $5021.88^{* *}$ & $6.19^{* *}$ & $8.18^{*}$ \\
Error & 48 & 124.80 & 28.36 & 2.21 & 4.12 \\
CV $(\%)$ & & 4.48 & 16.39 & 25.47 & 10.81 \\
\hline
\end{tabular}

Note. $* \mathrm{P}<0.05 ; * * \mathrm{P}<0.01$.

The most desirable trait in roselle is fiber yield, which is influenced by large values of plant height and basal diameter, high branching points, and low values of branch number (Maiti and Chakravarty, 1977). Table 4 shows the means of the 36 accessions and their rank in terms of potential fiber yield where HA-11, HA-07, HA-21, HA-33, and HA-12 came up as the top five accessions. Accession HA-11 exhibited the highest plant height $(355.88 \pm 25.66 \mathrm{~cm})$ a large value of height at first branching $(130.42 \pm 29.61 \mathrm{~cm})$, fewest branch number $(2.63 \pm 1.10)$ and the largest basal diameter $(23.32 \pm 4.11 \mathrm{~cm})$. Accession HA-07 ranked second with plant height $347.17 \pm 14.94 \mathrm{~cm}$, highbranching point at $143.52 \pm 27.18 \mathrm{~cm}$ above ground, few branches $3.78 \pm 1.59$ and large basal diameter of $20.52 \pm 2.20 \mathrm{~cm}$. Accession HA-21was tall $339.92 \pm 21.84 \mathrm{~cm}$, highest branching point at $157.08 \pm 32.97 \mathrm{~cm}$ above ground, few branch number of $4.21 \pm 1.53$ and a large basal diameter of $20.01 \pm 2.98 \mathrm{~cm}$. Accessions HA-33, and HA-12 with plant height, height at first branching, branch number, and basal diameter of $334.78 \pm 29.40 \mathrm{~cm}, 137.13 \pm 25.53 \mathrm{~cm}, 4.48 \pm 1.62,19.62 \pm 1.56 \mathrm{~cm}$ and $324.85 \pm 29.93 \mathrm{~cm}, 144.78 \pm 35.11 \mathrm{~cm}$, $5.22 \pm 1.97$ and $21.05 \pm 1.83 \mathrm{~cm}$, respectively, ranked fourth and fifth in fiber yield potential. The remaining accessions exhibited relatively low mean values of plant height (214 to $249 \mathrm{~cm})$, high mean branch number (5 to 8 ) at low mean branching points $(8.59$ to $20.90 \mathrm{~cm})$, and smaller mean basal diameters of less than $20 \mathrm{~cm}$.

\subsection{Shannon-Wiener Diversity Index in H. sabdariffa var. altissima Accessions}

Variation within the roselle populations was assessed by Shannon-Weiner Diversity Index (SDI) on the quantitative traits. Mean SDI of the 36 populations was $0.63 \pm 0.34$ with a range of 0.00 to 1.00 . The SDI values were very different among the traits. Largest variation resided in branch number $0.83 \pm 0.12$, basal diameter 
$0.80 \pm 0.11$, and plant height $0.75 \pm 0.16$, whereas height at first branching was low at $0.13 \pm 0.31$. Mean SDI values of individual accessions were high and ranged from 0.42 to 0.86 . All accessions had mean SDI values above 0.50 except HA-10 which had a value of 0.42 (Table 5). Majority of the SDI values occurred within the ranges of 0.50-0.59 (13) and 0.60-0.69 (17) (Figure 2). Accessions with high SDI values were HA-07 (0.79), HA-11(0.79), HA-12 (0.86), HA-21 (0.76), and HA-33 (0.79).

Table 3. Mean, standard deviation, minimum, and maximum traits evaluated in 36 H. sabdariffa L. var. altissima accessions originating from 18 districts in Ghana in 2016

\begin{tabular}{|c|c|c|c|c|c|}
\hline No. & District & Plant height $(\mathrm{cm})$ & Height at first branching $(\mathrm{cm})$ & Branch number & Basal diameter $(\mathrm{mm})$ \\
\hline 1 & Bawku Municipal & $220.63 \pm 31.98(188-285)$ & $14.28 \pm 7.36(4.3-29.4)$ & $5.5 \pm 3.12(1-9)$ & $15.17 \pm 4.21(10.13-21.2)$ \\
\hline 2 & Bolgatanga Municipal & $251.46 \pm 41.72(191-380)$ & $33.63 \pm 47.25(1.2-200)$ & $6.03 \pm 3.23(1-17)$ & $20.61 \pm 2.37(17-24.18)$ \\
\hline 3 & Ejisu-Juaben & $231.67 \pm 12.72(206-248)$ & $10.82 \pm 6.88(3.7-34.4)$ & $3.76 \pm 1.73(1-8)$ & $19.44 \pm 3.97(15.16-26.24)$ \\
\hline 4 & Ejura-Sekyedumase & $229.54 \pm 23.96(199-289)$ & $13.73 \pm 6.64(2.5-32.6)$ & $4.42 \pm 1.86(2-9)$ & $18.26 \pm 3.26$ 12.38-24.3) \\
\hline 5 & Garu-Tempane & $242.40 \pm 23.25(207-291)$ & $11.10 \pm 4.56(2.6-20)$ & $6.65 \pm 2.96(1-13)$ & $18.73 \pm 3.77(10.12-25.24)$ \\
\hline 6 & Gowri-Yorogo & $214.86 \pm 19.72(187-255)$ & $11.41 \pm 3.99(2.4-17.1)$ & $6.57 \pm 1.83(5-10)$ & $19.42 \pm 1.83(16.14-22.31)$ \\
\hline 7 & Kassena Nankana & $266.30 \pm 58.94(164-389)$ & $45.00 \pm 54.78(1.5-200)$ & $6.46 \pm 3.43(1-14)$ & $21.28 \pm 4.60(11.27-30.15)$ \\
\hline 8 & Kintampo Municipal & $228.83 \pm 22.26(193-263)$ & $10.8 \pm 4.56(3.7-19.5)$ & $6.33 \pm 2.46(3-11)$ & $21.57 \pm 2.79(15-26.3)$ \\
\hline 9 & Kumasi & $234.36 \pm 14.02(203-268)$ & $12.84 \pm 6.38(1.7-26.4)$ & $6.1 \pm 3.23(1-15)$ & $18.22 \pm 2.9(12.42-26.05)$ \\
\hline 10 & Mampong Municipal & $219.50 \pm 11.60(202-234)$ & $17.39 \pm 6.45$ 9.9-28.2) & $4.25 \pm 2.43(1-8)$ & $13.51 \pm 3.10(10.47-19.47)$ \\
\hline 11 & Sagnarigu-Gariba & $248.64 \pm 16.97(212-280)$ & $15.06 \pm 5.93(6.3-23)$ & $8.27 \pm 2.10(5-11)$ & $18.22 \pm 2.77(12.13-25.44)$ \\
\hline 12 & Savelugu-Nanton & $308.27 \pm 48.91(229-387)$ & $107.19 \pm 64.66(5.9-215)$ & $4.69 \pm 2.03(1-10)$ & $20.06 \pm 2.30(14.14-24.4)$ \\
\hline 13 & Sissala & $221.37 \pm 15.06(175-250)$ & $14.30 \pm 6.18(2.9-32)$ & $4.81 \pm 3.79(1-20)$ & $17.41 \pm 3.92(11.13-31.34)$ \\
\hline 14 & Talensi & $225.46 \pm 16.05(202-258)$ & $13.58 \pm 5.69(1.9-27)$ & $4.88 \pm 3.33(1-14)$ & $16.47 \pm 2.94(11.26-22.32)$ \\
\hline 15 & Tamale Metropolitan & $233.11 \pm 16.90(217-290)$ & $14.92 \pm 6.68(5.9-27.2)$ & $5.44 \pm 1.79(3-8)$ & $17.53 \pm 3.12(11.43-22.31)$ \\
\hline 16 & Techiman Municipal & $287.26 \pm 58.26(198-378)$ & $88.84 \pm 75.97(3.6-211)$ & $4.61 \pm 2.65(1-14)$ & $19.38 \pm 2.62(15.3-26.07)$ \\
\hline 17 & Wa Municipal & $241.95 \pm 12.01(222-269)$ & $15.24 \pm 4.5(9.3-23.4)$ & $4.42 \pm 1.74(2-8)$ & $17.56 \pm 1.89(15.16-21.47)$ \\
\hline \multirow[t]{2}{*}{18} & West Mamprusi & $240.12 \pm 18.50(212-286)$ & $13.54 \pm 4.78(8.1-25)$ & $5.80 \pm 1.80(2-10)$ & $17.91 \pm 2.23(13.26-21.34)$ \\
\hline & Mean square & $2,344.49^{*}$ & $3,096.44 *$ & 4.41 & 7.03 \\
\hline
\end{tabular}

Note. $* \mathrm{P}<0.05$.

Collectively, the accessions in the districts exhibited intermediate to high diversities. Savelugu-Nanton recorded the highest SDI value $0.73 \pm 0.2$ arising from the large diversity in plant height $(0.75$ to 0.93$)$, branch number ( 0.84 to 0.94$)$, and basal diameter $(0.61$ to 0.83$)$ only. Diversity in height at first branching was minimal except in HA-07 and HA-12, which gave substantial values 0.77 and 0.93 , respectively. Other districts with high SDI values between 0.60 and 0.70 were Bolgatanga Municipal (0.63 \pm 0.34$)$, Ejisu-Juaben (0.62 \pm 0.42$)$, Ejura-Sekyedumase $(0.64 \pm 0.42)$, Garu-Tempane (0.63 \pm 0.42$)$, Gowri-Yorogo $(0.67 \pm 0.45)$, Kassena-Nankana $(0.63 \pm 0.27)$, Kintampo Municipal (0.62 \pm 0.43$)$, Mampong Municipal (0.62 \pm 0.43$)$, Talensi $(0.67 \pm 0.45)$, Tamale Metropolitan (0.68 \pm 0.46$)$, and Wa Municipal $(0.66 \pm 0.45)$. 
Table 4. Mean, standard deviation, minimum, maximum values of traits of $36 \mathrm{H}$. sabdariffa $\mathrm{L}$. var. altissima accessions evaluated in Ghana in 2016 and their rank based on potential fiber yield

\begin{tabular}{|c|c|c|c|c|c|}
\hline $\mathrm{ACC}$ & Plant height $(\mathrm{cm})$ & Height at first branch $(\mathrm{cm})$ & Branch number & Basal diameter $(\mathrm{mm})$ & Rank \\
\hline HA-01 & $241.95 \pm 12.01(222-269)$ & $15.24 \pm 4.50(9.3-23.4)$ & $4.42 \pm 1.74(2-8)$ & $17.56 \pm 1.89(15.16-21.47)$ & 12 \\
\hline HA-02 & $228.83 \pm 22.26(193-263)$ & $10.80 \pm 4.56(3.7-19.5)$ & $6.33 \pm 2.46(3-11)$ & $21.57 \pm 2.79(15-26.3)$ & 24 \\
\hline HA-03 & $248.64 \pm 16.97(212-280)$ & $15.06 \pm 5.93(6.3-23)$ & $8.27 \pm 2.10(5-11)$ & $20.61 \pm 2.37(17-24.18)$ & 8 \\
\hline HA-04 & $228.29 \pm 9.43(203-243)$ & $13.27 \pm 5.09(3.5-22.4)$ & $4.57 \pm 1.72(2-8)$ & $18.75 \pm 1.87(15.29-22.41)$ & 25 \\
\hline HA-05 & $248.05 \pm 20.73(210-279)$ & $20.90 \pm 21.38(6-11)$ & $7.00 \pm 3.01(2-14)$ & $17.56 \pm 2.39(11.30-22.03)$ & 9 \\
\hline HA-06 & $220.63 \pm 31.98(188-285)$ & $14.28 \pm 7.36(4.3-29.4)$ & $5.50 \pm 3.12(1-9)$ & $15.17 \pm 4.21(10.13-21.2)$ & 31 \\
\hline HA-07 & $347.17 \pm 14.94(331-382)$ & $143.52 \pm 27.18(104-205)$ & $3.78 \pm 1.59(2-7)$ & $20.52 \pm 2.20(14.14-24.23)$ & 2 \\
\hline HA-08 & $230.89 \pm 13.53(213-259)$ & $18.32 \pm 5.21(8.7-27.3)$ & $5.94 \pm 2.21(3-10)$ & $18.78 \pm 2.53(14.08-22.31)$ & 21 \\
\hline HA-09 & $238.80 \pm 20.16(217-290)$ & $11.37 \pm 6.61(5.9-27.2)$ & $5.40 \pm 1.71(3-8)$ & $16.13 \pm 2.92(11.43-20.26)$ & 19 \\
\hline HA-10 & $229.82 \pm 11.16(198-248)$ & $14.39 \pm 6.96(3.6-25.3)$ & $5.05 \pm 3.48(1-14)$ & $18.70 \pm 2.01(15.3-24.12)$ & 23 \\
\hline HA-11 & $355.88 \pm 25.66(248-389)$ & $130.42 \pm 29.61(93-200)$ & $2.63 \pm 1.10(1-5)$ & $23.32 \pm 4.11(17.09-30.15)$ & 1 \\
\hline HA-12 & $324.85 \pm 29.93(264-387)$ & $144.78 \pm 35.11(93-215)$ & $5.22 \pm 1.97(2-10)$ & $21.05 \pm 1.83(18.35-24.4)$ & 5 \\
\hline HA-13 & $239.05 \pm 14.04(213-260)$ & $16.52 \pm 5.70(6.10-25.4)$ & $6.48 \pm 3.92(2-13)$ & $18.70 \pm 3.50(14.03-25.44)$ & 18 \\
\hline HA-14 & $224.17 \pm 14.47(193-252)$ & $14.02 \pm 13.95(5.4-77)$ & $6.50 \pm 4.05(1-17)$ & $17.50 \pm 2.55(12.13-22.7)$ & 28 \\
\hline HA-15 & $249.19 \pm 12.87(212-271)$ & $15.10 \pm 7.96(4.6-40)$ & $4.38 \pm 2.46(1-10)$ & $18.78 \pm 2.86(13.28-23.41)$ & 7 \\
\hline HA-16 & $243.84 \pm 16.60(210-267)$ & $14.11 \pm 7.10(4.8-30.2)$ & $7.00 \pm 2.81(3-12)$ & $18.20 \pm 2.77(12.26-23.11)$ & 10 \\
\hline HA-17 & $206.89 \pm 8.65(199-223)$ & $11.62 \pm 5.95(2.5-22.7)$ & $6.00 \pm 2.00(3-9)$ & $19.62 \pm 1.49(17.46-22.31)$ & 35 \\
\hline HA-18 & $239.83 \pm 14.86(218-268)$ & $14.10 \pm 5.94(3.7-26.4)$ & $6.35 \pm 3.46(2-15)$ & $17.09 \pm 3.03(12.42-26.05)$ & 17 \\
\hline HA-19 & $231.67 \pm 12.72(206-248)$ & $10.82 \pm 6.88(3.7-34.4)$ & $3.76 \pm 1.73(1-8)$ & $19.44 \pm 3.97(15.16-26.24)$ & 20 \\
\hline HA-20 & $241.53 \pm 20.45(204-289)$ & $14.85 \pm 6.88(5.7-32.6)$ & $3.59 \pm 1.12(2-6)$ & $17.53 \pm 3.72(12.38-24.3)$ & 13 \\
\hline HA-21 & $339.92 \pm 21.84(309-378)$ & $157.08 \pm 32.97(104-211)$ & $4.21 \pm 1.53(1-8)$ & $20.01 \pm 2.98(16.17-26.07)$ & 3 \\
\hline HA-22 & $240.12 \pm 18.50(212-286)$ & $13.54 \pm 4.78(8.10-25)$ & $5.80 \pm 1.80(2-10)$ & $17.91 \pm 2.23(13.26-21.34)$ & 16 \\
\hline HA-23 & $225.46 \pm 16.05(202-258)$ & $13.58 \pm 5.69(1.90-27)$ & $4.88 \pm 3.33(1-14)$ & $16.47 \pm 2.94(11.26-22.32)$ & 27 \\
\hline HA-24 & $206.50 \pm 16.87(164-236)$ & $8.67 \pm 5.19(1.7-17.3)$ & $8.89 \pm 2.19(6-13)$ & $17.19 \pm 3.75(11.27-24.25)$ & 36 \\
\hline HA-25 & $240.67 \pm 17.91(198-265)$ & $12.82 \pm 7.37(1.2-28.2)$ & $5.67 \pm 3.62(2-13)$ & $16.47 \pm 2.18(13.43-20.22)$ & 15 \\
\hline HA-26 & $230.82 \pm 14.20(214-250)$ & $12.75 \pm 1.56(10.7-15.4)$ & $7.73 \pm 1.56(5-11)$ & $16.83 \pm 1.09(15.22-19.14)$ & 22 \\
\hline HA-27 & $249.76 \pm 12.04(228-277)$ & $13.04 \pm 5.11(1.5-22.8)$ & $7.76 \pm 3.83(1-13)$ & $19.15 \pm 3.66(11.33-25.4)$ & 6 \\
\hline HA-28 & $219.50 \pm 11.60(202-234)$ & $17.39 \pm 6.45(9.9-28.2)$ & $4.25 \pm 2.43(1-8)$ & $13.51 \pm 3.10(9.47-19.47)$ & 32 \\
\hline HA-29 & $217.24 \pm 16.81(175-250)$ & $12.53 \pm 4.98(6-19.7)$ & $7.06 \pm 5.03(1-20)$ & $20.21 \pm 4.62(11.13-31.34)$ & 33 \\
\hline HA-30 & $224.08 \pm 13.45(204-248)$ & $15.35 \pm 6.67(2.9-32)$ & $3.35 \pm 1.52(1-7)$ & $15.58 \pm 1.82(12.05-20.48)$ & 29 \\
\hline HA-31 & $242.40 \pm 23.25(207-291)$ & $11.10 \pm 4.56(2.6-20)$ & $6.65 \pm 2.96(1-13)$ & $18.73 \pm 3.77(10.12-25.24)$ & 11 \\
\hline HA-32 & $222.05 \pm 18.65(191-280)$ & $9.98 \pm 5.18(3-18.1)$ & $6.09 \pm 2.96(1-11)$ & $17.80 \pm 2.84(12.28-24.23)$ & 30 \\
\hline HA-33 & $334.78 \pm 29.40(248-380)$ & $137.13 \pm 25.53(103-200)$ & $4.48 \pm 1.62(2-8)$ & $19.62 \pm 1.56(16.38-23.18)$ & 4 \\
\hline HA-34 & $214.86 \pm 19.72(187-255)$ & $11.41 \pm 3.99(2.4-17.1)$ & $6.57 \pm 1.83(5-10)$ & $19.42 \pm 1.83(16.14-22.31)$ & 34 \\
\hline HA-35 & $241.15 \pm 15.38(229-280)$ & $14.66 \pm 4.82(5.9-23.8)$ & $5.00 \pm 2.27(1-10)$ & $18.20 \pm 1.95(15.31-22.24)$ & 14 \\
\hline HA-36 & $226.50 \pm 7.89(210-239)$ & $8.59 \pm 5.67(1.7-23.4)$ & $5.75 \pm 2.93(1-11)$ & $19.11 \pm 2.31(13.31-23.41)$ & 26 \\
\hline
\end{tabular}


Table 5. Shannon Weiner Diversity Index of morphological evaluation of $36 \mathrm{H}$. sabdariffa L. var. altissima accessions from 18 Districts conducted in Ghana in 2016

\begin{tabular}{|c|c|c|c|c|c|c|c|c|}
\hline Accession & District & $\begin{array}{l}\text { Plant } \\
\text { height }(\mathrm{cm})\end{array}$ & $\begin{array}{l}\text { Height at first } \\
\text { branching }(\mathrm{cm})\end{array}$ & Branch number & $\begin{array}{l}\text { Basal } \\
\text { diameter }(\mathrm{mm})\end{array}$ & $\begin{array}{l}\text { Accession } \\
\text { mean }\end{array}$ & $\begin{array}{l}\text { District } \\
\text { mean }\end{array}$ & $\begin{array}{l}\text { Rank in } \\
\text { diversity }\end{array}$ \\
\hline HA-06 & Bawku Municipal & 0.63 & 0.00 & 0.89 & 0.63 & $0.53 \pm 0.38$ & $0.53 \pm 0.38$ & 12 \\
\hline HA-13 & \multirow{7}{*}{ Bolgatanga Municipal } & 0.96 & 0.00 & 0.97 & 0.78 & $0.68 \pm 0.46$ & \multirow{7}{*}{$0.63 \pm 0.34$} & \multirow{7}{*}{6} \\
\hline HA-14 & & 0.54 & 0.00 & 0.75 & 0.83 & $0.53 \pm 0.37$ & & \\
\hline HA-15 & & 0.65 & 0.00 & 0.99 & 0.83 & $0.62 \pm 0.43$ & & \\
\hline HA-16 & & 0.96 & 0.00 & 0.83 & 0.59 & $0.59 \pm 0.43$ & & \\
\hline HA-25 & & 0.76 & 0.00 & 0.91 & 0.99 & $0.66 \pm 0.45$ & & \\
\hline HA-32 & & 0.42 & 0.00 & 0.94 & 0.69 & $0.51 \pm 0.40$ & & \\
\hline HA-33 & & 0.54 & 0.94 & 0.85 & 0.85 & $0.79 \pm 0.17$ & & \\
\hline HA-19 & Ejisu-Juaben & 0.92 & 0.00 & 0.85 & 0.71 & $0.62 \pm 0.42$ & $0.62 \pm 0.42$ & 7 \\
\hline HA-17 & \multirow{2}{*}{ Ejura-Sekyedumase } & 0.81 & 0.00 & 0.89 & 0.81 & $0.63 \pm 0.42$ & \multirow{2}{*}{$0.64 \pm 0.42$} & \multirow{2}{*}{5} \\
\hline HA-20 & & 0.82 & 0.00 & 0.86 & 0.87 & $0.64 \pm 0.43$ & & \\
\hline HA-31 & Garu-Tempane & 0.85 & 0.00 & 0.83 & 0.83 & $0.63 \pm 0.42$ & $0.63 \pm 0.42$ & 6 \\
\hline $\mathrm{HA}-34$ & Gowri-Yorogo & 0.84 & 0.00 & 0.99 & 0.85 & $0.67 \pm 0.45$ & $0.67 \pm 0.45$ & 3 \\
\hline HA-05 & \multirow{5}{*}{ Kassena-Nankana } & 0.75 & 0.27 & 0.62 & 0.62 & $0.56 \pm 0.21$ & \multirow{5}{*}{$0.63 \pm 0.27$} & \multirow{5}{*}{6} \\
\hline HA-11 & & 0.61 & 0.86 & 0.74 & 0.93 & $0.79 \pm 0.14$ & & \\
\hline HA-24 & & 0.81 & 0.00 & 0.78 & 0.64 & $0.56 \pm 0.38$ & & \\
\hline HA-26 & & 0.95 & 0.00 & 0.72 & 0.99 & $0.66 \pm 0.46$ & & \\
\hline $\mathrm{HA}-27$ & & 0.67 & 0.00 & 0.85 & 0.85 & $0.59 \pm 0.40$ & & \\
\hline HA- 02 & Kintampo Municipal & 0.92 & 0.00 & 0.66 & 0.89 & $0.62 \pm 0.43$ & $0.62 \pm 0.43$ & 7 \\
\hline HA-04 & \multirow{3}{*}{ Kumasi } & 0.59 & 0.00 & 0.94 & 0.72 & $0.56 \pm 0.40$ & \multirow{3}{*}{$0.56 \pm 0.37$} & \multirow{3}{*}{11} \\
\hline HA-18 & & 0.99 & 0.00 & 0.55 & 0.78 & $0.58 \pm 0.43$ & & \\
\hline HA-36 & & 0.67 & 0.00 & 0.65 & 0.83 & $0.54 \pm 0.37$ & & \\
\hline HA-28 & Mampong Municipal & 0.67 & 0.00 & 0.91 & 0.91 & $0.62 \pm 0.43$ & $0.62 \pm 0.43$ & 7 \\
\hline HA-03 & Sagnarigu-Gariba & 0.49 & 0.00 & 0.91 & 0.83 & $0.56 \pm 0.41$ & $0.56 \pm 0.41$ & 11 \\
\hline HA-07 & \multirow{4}{*}{ Savelugu-Nanton } & 0.93 & 0.77 & 0.84 & 0.61 & $0.79 \pm 0.14$ & \multirow{4}{*}{$0.73 \pm 0.21$} & \multirow{4}{*}{1} \\
\hline HA-08 & & 0.85 & 0.00 & 0.93 & 0.79 & $0.64 \pm 0.43$ & & \\
\hline HA-12 & & 0.84 & 0.93 & 0.87 & 0.82 & $0.86 \pm 0.05$ & & \\
\hline HA-35 & & 0.75 & 0.00 & 0.94 & 0.83 & $0.63 \pm 0.43$ & & \\
\hline HA-29 & \multirow{2}{*}{ Sissala } & 0.55 & 0.00 & 0.84 & 0.85 & $0.56 \pm 0.40$ & \multirow{2}{*}{$0.58 \pm 0.42$} & \multirow{2}{*}{9} \\
\hline HA-30 & & 0.50 & 0.00 & $\begin{array}{c}0.99 \\
-\end{array}$ & 0.91 & $0.60 \pm 0.45$ & & \\
\hline HA-23 & Talensi & 0.92 & 0.00 & 0.94 & 0.83 & $0.67 \pm 0.45$ & $0.67 \pm 0.45$ & 3 \\
\hline HA-09 & Tamale Metropolitan & 0.82 & 0.00 & 0.94 & 0.96 & $0.68 \pm 0.46$ & $0.68 \pm 0.46$ & 2 \\
\hline HA-10 & \multirow{2}{*}{ Techiman Municipal } & 0.59 & 0.00 & 0.48 & 0.59 & $0.42 \pm 0.28$ & \multirow{2}{*}{$0.59 \pm 0.09$} & \multirow{2}{*}{8} \\
\hline HA-21 & & 0.54 & 0.93 & 0.78 & 0.78 & $0.76 \pm 0.16$ & & \\
\hline HA-01 & Wa Municipal & 1.00 & 0.00 & 0.76 & 0.87 & $0.66 \pm 0.45$ & $0.66 \pm 0.45$ & 4 \\
\hline HA-22 & West Mamprusi & 0.79 & 0.00 & 0.78 & 0.69 & $0.57 \pm 0.38$ & $0.57 \pm 0.38$ & 10 \\
\hline Mean & & $0.75 \pm 0.16$ & $0.13 \pm 0.31$ & $0.83 \pm 0.12$ & $0.80 \pm 0.11$ & & & \\
\hline
\end{tabular}




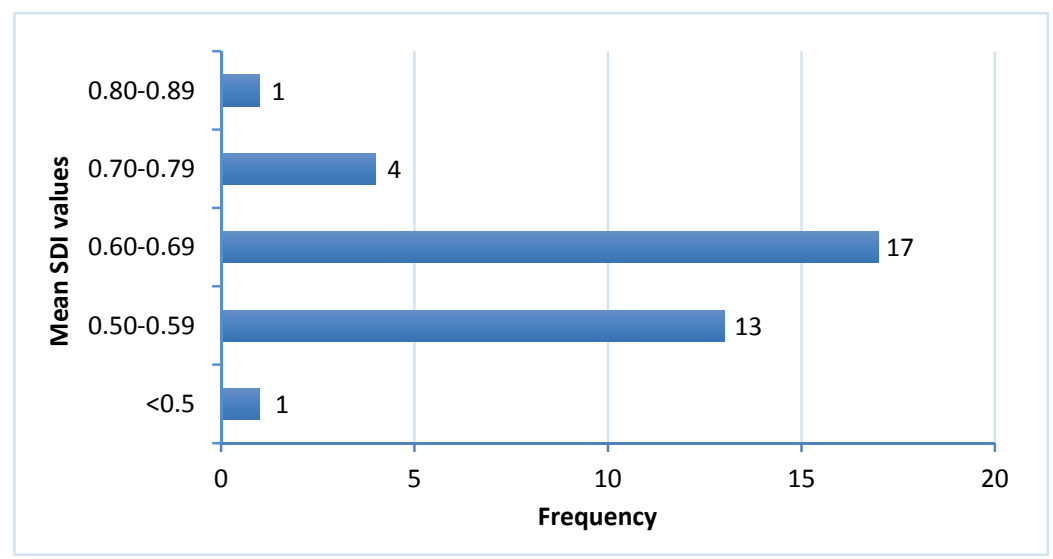

Figure 2. Frequency of Shannon Weiner Diversity Index among 36 roselle fiber accessions evaluated in Ghana in 2016

\section{Discussion}

Roselle fiber type is an important commodity that is marketed around the globe for its multipurpose bast fiber. A recent interest in roselle fiber in Ghana for economic expoitation necessitates its evaluation to identify important economic traits, useful accessions of commercial value, and to estimate the genetic diversity therein for genetic improvement and conservation.

The unequal distribution of the accessions in the study area indicates that roselle fiber type is not very well known among the localities compared to the more common vegetable type, except for the indigenous folks who make tethers from roselle for their livestock. Rapid rate of urbanization in the northern sector of Ghana may have contributed to lack of knowledge of this germplasm. Additionally, seed morphology for the fiber and vegetable roselle are similar, and information on morphological differences in the seeds is not available, so that roselle fiber type seed collection is fraught with challenges. An informed and well-planned seed collection by a team of taxonomists and the indigenous people should provide a more accurate roselle fiber type germplasm collection and this is urgently needed.

The large variability in plant type, branching habit, and leaf shape were also reported by Alam et al. (2006) among four Bangladesh Hibiscus species, the H. sabdariffa, H. cannabinus, H. acetosella and H. radiatus. Plants having uniformly green stems, few branching and high branching points are more desirable for fiber production than pigmented, extensively branched and low branching types. Youngberg (1929) confirmed a 5\% yield advantage in green over pigmented roselle. The indigenous folks testify that they extract fiber from green roselle but not from the pigmented genotypes for claim of good fiber yield and quality. This claim needs validation through a scientific research program. The less variable height-at-first-branching, stem pubescence, leaf colour, leaf size, and stem pubescence appeared to be traits that are conserved in the bast fiber plants of Hibiscus species, including H. sabdariffa variety sabdariffa (Coffie, 2016; Siepe et al., 1997), H. cannabinus (Faruq et al., 2013, and $H$. vulgaris, $H$. rosens, $H$. mutabilis (Siepe et al., 1997).

Large variability in plant height, branch number, and basal diameter were also identified in $H$. sabdariffa var. sabdariffa from West Africa (Coffie, 2016), Asia and Europe genotypes (Siepe et al., 1997), H. cannabinus accessions from Kenya, China, U.S.A. and Australia (Faruq et al., 2013). This large variability means that genetic improvement in these traits via selection is possible. The differences in the quantitative traits may be attributed to varying genotypic differences. A plant height of 164 to $389 \mathrm{~cm}$ falls within the range of plant height of $H$. sabdariffa var. altissima which can grow from 250 to $500 \mathrm{~cm}$ and is usually branchless (Eltayeib and Elaziz, 2014). Plant height and branching in roselle is governed by day length and temperature and its effect on flowering (Dempsey, 1975; Tindall, 1983). Roselle is ambiphotoperiodic whereby it flowers in short days and also in extremely long days (Mansour, 1975). Once roselle plants flower, vegetative growth slows down. Because roselle is used for fiber and paper pulp, the length and thickness of the stem determine fiber yield. Short stems arising from early flowering or poor nutrition and irrigation limit yield. A short day length of $8 \mathrm{~h}$ promotes more flowering, many branches, short plant height, and shallow tri-lobed leaves with upper slender leaves, whereas long days of 16-h light induces vegetative growth with few branches, tall plants, and deeply lobed leaves. The climate in the study site consisted of $12 \mathrm{~h} 30 \mathrm{~min}$ in July 2016 which reduced to $11 \mathrm{~h} 44 \mathrm{~min}$ in 
December 2016. Temperature ranged from $22{ }^{\circ} \mathrm{C}$ to $31{ }^{\circ} \mathrm{C}$, a typical intermediate day length (World Weather and Climate Information, 2016). Although 106 plants were tall, only two accessions (HA-07 and HA-11) had few branches, while 188 plants had intermediate height with few branches. On the basis of tall plant height and large basal diameter, high but few branching the most important traits for selection of genotypes for bast fiber production and quality include HA-07, HA-11, HA-12, HA-21, and HA-33 from Techiman and Bolgatanga Municipal, Kassena-Nankana, and Savelugu-Nanton districts in the Brong Ahafo, Northern and Upper East regions of Ghana. These accessions had plant height in excess of $250 \mathrm{~cm}$, basal diameter greater than $20 \mathrm{~mm}$, maximum of four branches at branching heights exceeding $130 \mathrm{~cm}$. Low branching points and extensive branching interrupt the fiber strands and reducs fineness. Maiti and Chakravarty (1977) indicated that the strong environmental influence on plant height and basal diameter makes prediction and selection for fiber yield a complicated task in roselle, however, an additional parameter, which is the length of the vegetative growth period highlights the plant's economic value for fiber. On the basis of diversity index and fiber yield characteristics, roselle hotspots in northern Ghana include Savelugu-Nanton, Tamale Metropolitan, Gowri-Yorogo and Talensi districts. Other districts of large diversity include Wa Municpal and Ejura-Sekyedumase. A more robust and well-planned collection of accessions from all the hot spot regions in northern Ghana is needed. In addition, evaluation of roselle accessions in their collection sites would be required to ascertain a genotype by environment interaction component that contributes to the variability. The fairly high variability observed among the $H$. sabdariffa var. altissima accessions in this study was unexpected as roselle plant is self-pollinating. The environment and most probably mutation that had accrued over several generations may have contributed to the observed variability. To the best of our knowledge, this is the first report on $H$. sabdariffa var. altissima diversity in Ghana.

\section{Conclusion}

In conclusion, a large variability resides in the roselle accessions of northern Ghana. This variability is indicative of a rich diversity which may be harnessed for improvement in economic traits such as fiber yield and quality. The differences in the diversity among the districts would be beneficial to a systematic collection of roselle in northern Ghana. .

\section{Acknowledgements}

The authors want to acknowledge the Kwame Nkrumah University of Science and Technology for providing the research field for the study.

\section{References}

Alam, N., Pasha, M. K., \& Ahmed, S. (2006). Identification of some Hibiscus germplasm through numerical analysis. Bangladesh Journal of Plant Taxonomy, 13, 49-54. https://doi.org/10.3329/bjpt.v13i1.593

Andrews, F. W. (1952). Flowering Plants of the Anglo-Egyptian Sudan (pp. 28-30). Buncle and Company Limited, Arbroath, Scotland.

Berger, W. H., \& Parker, F. L. (1970). Diversity of planktonic foraminifera in deep-sea sediments. Science, 168, 1345-134. https://doi.org/10.1126/science.168.3937.1345

Boulanger, J., Follin, J. C., \& Bourely, J. (1984). Les hibiscus textiles en Afrique tropicale, $1^{\text {ere }}$ partie: Conditions particulières de production du kenaf et de la roselle (5th ed.). Coton et Fibres Tropicales.

Brower, J. E., \& Zar, J. H. (1977). Field and Laboratory Methods for General Ecology. C. Brown Company Publishers. Boston, MA.

Chand, N., Vishwaama, S. R., Verma, O. P., \& Manoj, K. (2008). Worth of genetic parameters to sort out new elite barely lines over heterogeneous environments. Barley Genetics Newsletter, 38, 10-13.

Coffie, N. (2016). Characterization of roselle (Hibiscus sabdariffa L.) accessions by agro-morphological and RAPD genotyping (MPhil Thesis, Department of Biochemistry and Biotechnology, Kwame Nkrumah University of Science and Technology, Kumasi, Ghana).

DeJong, T. M. (1975). A comparison of three diversity indices based on their components of richness and evenness. Oikos, 26, 222-227. https://doi.org/10.2307/3543712

Dempsey, J. M. (1975). Fiber Crops (pp. 203-233). Rose Printing Company, Tallahassee, FL.

Dudley, J. W., \& Moll, R. H. (1969). Interpretation and use of estimates of heritability and genetic variances in plant breeding. Crop Science, 9, 257-262. https://doi.org/10.2135/cropsci1969.0011183X000900030001x 
Eltayeib, A. A., \& Elaziz, A. A. (2014). Physiochemical properties of Roselle (Hibiscus sabdariffa L.) seeds oil in North Kordofan, Sudan. Journal of Scientific and Innovative Research, 3, 578-582.

Faruq, G., Alamgir, M. A., Rahman, M. M., Motior, M. R., Zakaria, H. P., Marchalina, B., \& Mohamed, N. A. (2013). Morphological characterization of kenaf (Hibiscus cannabinus) in Malaysian tropical environment using multivariate analysis. Journal of Animal and Plant Sciences, 23, 60-67.

Gomez-Leyva, J. F., Acosta, L. A. M., Muraira, I. G. L., Espino, H. S., Ramirez-Cervantes, F., \& Andrade-Gonzalez, I. (2008). Multiple shoot regeneration of roselle (Hibiscus sabdariffa L.) from a shoot apex system. International Journal of Botany, 4, 326-330. https://doi.org/10.3923/ijb.2008.326.330

Hanboonsong, Y., Vinijsanun, T., \& Ponragdee, W. (2000). Molecular Characterization and Genetic Relationships of Roselle Germplasm in Thailand. Proceedings of the Final Workshop on Application of Biotechnology in the Improvement of Jute, Kenaf and Allied Fibers, China (pp. 95-106).

Imperial Institute, Great Britain. (1912). Hibiscus fibers from Northern territories, Gold Coast. Imperial Institute Bulletin, 10, 51-55.

Loya, Y. (1972). Community structure and species diversity of hermatypic corals at Eilat, Red Sea. Marine Biology, 13, 100-123. https://doi.org/10.1007/BF00366561

Maiti, R. K., \& Chakravarty, K. (1977). A comparative study on yield components and quality of Indian bast fibers. Economic Botany, 31, 55-60. https://doi.org/10.1007/BF02860653

Mansour, B. M. M. (1975). Effects of temperature and day length on growth and flowering of roselle, Hibiscus sabdariffa L. Scientia Horticulturae, 3, 129-135. https://doi.org/10.1016/0304-4238(75)90018-7

May, R. M. (1975). Patterns of species abundance and diversity. In M. L. D. Cody, \& J. M. Diamond (Eds.), Ecology and Evolution of Communities (pp. 81-120). Harvard University Press, Cambridge, Massachussetts.

McIntosh, R. P. (1967). An index of diversity and the relation of certain concepts to diversity. Ecology, 48, 1115-1126. https://doi.org/10.2307/1932674

Medagam, T. R., Begum, H., Rao, N. H., Neelam, S., Pandravada, S. R., \& Natarajan, S. (2015). Genetic diversity and variability studies in landraces for key agro-economic traits in vegetable Roselle (Hibiscus sabdariffa var. sabdariffa L.). Jordan Journal of Biological Sciences, 8, 113-125. https://doi.org/10.12816/ 0027557

Morton, J. F. (1987). Roselle: Fruits of Warm Climates (pp. 281-286). Greensboro, NC: Media, Inc.

Omalsaad, A. K. M., Islam, A., Juhan, M. A., Yaakob, Z., \& Osman, M. (2014). Genetic relationship between roselle (Hibiscus sabdariffa L.) and kenaf (Hibiscus cannabinus L.) accessions through optimization of PCR based RAPD method. Emirates Journal of Food Agriculture, 26, 274-258. https://oi.org/10.9755/ ejfa.v26i3.16498

Perry, L. M. (1980). Medicinal Plants of East and Southeast Asia (p. 620). Cambridge, MA: MIT Press.

Pielou, E. C. (1969). An Introduction to Mathematical Ecology. New York: Wiley-Interscience.

Rhodin, E. G., \& Panchoo, L. (1990). Effect of transplant timing and terminal bud removal on Sorrel. Caribbean Food Crop Society Proceedings, 26, 245-254.

Risser, P. G., \& Rice, E. L. (1971). Diversity in tree species in Oklahoma upland forests. Ecology, 52, 876-880. https://doi.org/10.2307/1936036

SAS Institute Inc. (2011). Base SAS® 9.3 Procedures Guide: Statistical Procedures. SAS Institute, Cary, North Carolina.

Shannon, C. E., \& Weaver, W. (1949). The Mathematical Theory of Communication. University of Illinois Press, Urbana.

Siepe, T., Ventrella, D., \& Lapenta, E. (1997). Evaluation of genetic variability in a collection of Hibiscus cannabinus and Hibiscus spp. Industrial Crops and Products, 6, 343-352. https://doi.org/10.1016/ S0926-6690(97)00025-3

Simpson, E. H. (1949). Measurement of diversity. Nature, 1, 63-688. https://doi.org/10.1038/163688a0

Tindall, H. D. (1983). Vegetable in Tropics (p. 734). McMillan International College Edition, Hamshire. https://doi.org/10.1007/978-1-349-17223-8

Wester, P. J. (1912). Roselle, its cultivation and uses. Phillipine Agriculture Revolution, 5, 123-131. 
Wester, P. J. (1914). New varieties of roselle. Philippine Agricultural Review, 7, 266-269.

Whittaker, R. H. (1972). Evolution and measurement of species diversity. Taxonomy, 21, 213-251. https://doi.org/10.2307/1218190

World Weather and Climate Information. (2016). Climate: Average Monthly Weather in Kumasi, Ghana. Retrieved August 30, 2016, from https://weather-and-climate.com/average-monthly-rainfall-temperaturesunshine-kumasi/ghana

Youngberg, S. (1929). Annual report of the Bureau of Agriculture. Philippine Agriculture Revolution, 22, 89-102.

Yusof, M. M., \& Saud, H. M. (2009). Cultivars and variety identification and their genetic relationships by randomly amplified polymorphic DNA (RAPD) markers in roselle (Hibiscus sabdariffa L). Proceedings of the $8^{\text {th }}$ Congress on Genetics 4-6 August. Genting Highlands, Malaysia.

\section{Copyrights}

Copyright for this article is retained by the author (s), with first publication rights granted to the journal.

This is an open-access article distributed under the terms and conditions of the Creative Commons Attribution license (http://creativecommons.org/licenses/by/4.0/). 